\title{
SPACE SEXTIC CURVES WITH SIX BITANGENTS, AND SOME GEOMETRY OF THE DIAGONAL CUBIC SURFACE
}

\author{
by R. H. DYE \\ (Received 30th January 1995)
}

To W. L. Edge, active nonagenerian geometer

\begin{abstract}
A general space curve has only a finite number of quadrisecants, and it is rare for these to be bitangents. We show that there are irreducible rational space sextics whose six quadrisecants are all bitangents. All such sextics are projectively equivalent, and they lie by pairs on diagonal cubic surfaces. The bitangents of such a related pair are the halves of the distinguished double-six of the diagonal cubic surface. No space sextic curve has more than six bitangents, and the only other types with six bitangents are certain $(4,2)$ curves on quadrics. In the course of the argument we see that space sextics with at least six quadrisecants are either $(4,2)$ or $(5,1)$ quadric curves with infinitely many, or are curves which each lie on a unique, and non-singular, cubic sirface and have one half of a double-six for quadrisecants.
\end{abstract}

1991 Mathematics subject classification: Primary 14H50, Secondary 14J25.

\section{Introduction}

1.1. A non-planar non-singular irreducible curve in 3-dimensional complex projective space has, in general, a finite number of quadrisecants. If the curve has order $n$ and genus $g$ then their number is [7, p. 296], [12, p. 377]

$$
q=\frac{1}{12}(n-2)(n-3)^{2}(n-4)-\frac{1}{2} g\left(n^{2}-7 n+13-g\right) .
$$

To demand that one or more of these quadrisecants is a bitangent, having its four intersections with the curve as two coincident pairs, imposes extra conditions which are usually impossible to meet. Only exceptional curves possess bitangents, and their rarity makes them interesting. We should say, at once, that there are curves with an infinity of quadrisecants; for example, the intersection of a ruled surface with a surface of order at least four has the generators of the ruled surface for quadrisecants (at least). Then a finite number of these will touch the curve but, usually, none will touch it twice.

Space cubics and quartics have no quadrisecants. There is just one type of nonsingular space quintic with a finite positive number of quadrisecants; this is the quintic of genus 0 , which, by (1), has just one quadrisecant [12, p. 377]. It is possible for this quadrisecant to be a bitangent; take a rational normal quintic curve in 5-space, take the solid spanned by two of its skew tangent lines, and project from a general line in 
this solid so that both tangents project to a common bitangent. Interest thus turns to space sextics.

Non-singular space sextics can be [12, pp. 93, 393] of genus $0,1,2,3$ or 4 , when the respective values of $q$ are $6,3,1,0$ and 0 . One of the main purposes of this paper is to obtain a non-singular sextic $\mathscr{S}$ whose six quadrisecants are all bitangents (Section 2.1). In fact (Theorem 4) all such sextics are projectively equivalent. The group of self-projectivities of $\mathscr{S}$ is the alternating group $A_{5}$ (Theorem 4). A nonsingular space curve of order $n$ and genus $g$ has [2, p. 200], [12, p. 377] 4(n+3p-3) stalls, points where the tangent plane has more than its statutory minimum 3-point intersection. The 12 stalls of $\mathscr{S}$ are its contacts with its bitangents (Theorem 1). In the course of our discussion we shall see (Theorems 2,3) that any space sextic with infinitely many quadrisecants lies on a non-singular quadric, and that any such curve with at least six bitangents has exactly six, and is a $(4,2)$ curve on the quadric: by an $(\alpha, \beta)$ curve on the quadric we mean one of order $\alpha+\beta$ which meets each generator of one regulus of the quadric in $\alpha$ points, and each generator of the other in $\beta$ points; such a curve, if non-singular, has $[5$, p. 16], $[12$, pp. 82,122$]$ genus $(\alpha-1)(\beta-1)$. There are (Section 3.4) non-singular $(4,2)$ curves with six bitangents; they have genus 3 .

1.2 The six bitangents of $\mathscr{S}$ are one half of the distinguished double-six of a diagonal cubic surface $D$ (Theorem 1). A pentahedron $P$ of planes, no four concurrent, has ten edges and ten vertices. Each edge contains three vertices. Each vertex, common to three faces, is opposite to the edge common to the other two. The join of a vertex to a vertex on the opposite edge is a diagonal. Altogether $P$ has 15 diagonals, and there is a unique cubic surface, the non-singular diagonal surface $D$, containing them [6, p. 539], [9, pp. 199, 201]. $D$ has 12 other lines which form its distinguished double-six, $\delta[6$, p. 543], which may be displayed as

$$
\begin{array}{llllll}
a_{1} & a_{2} & a_{3} & a_{4} & a_{5} & a_{6} \\
b_{1} & b_{2} & b_{3} & b_{4} & b_{5} & b_{6}
\end{array}
$$

where each of these lines is skew to the other six lines in the same row or column, but meets the other five lines. The tangent plane to $D$ at a vertex of $P$ contains the three diagonals through that vertex; the ten vertices of $P$ are Eckardt points of $D[9$, p. 199]. If one takes the standard plane representation of a cubic surface by cubic curves through six points $A_{1}, A_{2}, \ldots, A_{6}$ not on a conic, then the neighbourhood of $A_{i}$ corresponds to the points of the line $a_{i}$ and the conic $\mathscr{B}_{i}$ through all the $A_{j}$ apart from $A_{i}$ corresponds to $b_{i}[1$, pp. 189-191], [6, p. 214], [7, pp. 480-489], [12, pp. 124-128]. Corresponding to the ten Eckardt points of $D$ are [4], [9, pp. 197, 214] ten Brianchon points of the hexagon $H$ with vertices $A_{1}, \ldots, A_{6}$, i.e. non-vertex points of concurrence of three of its edges. The geometry of $H$ was explored in [3]. In particular [3, p. 283], it was shown that there is a conic $\mathscr{C}$ that has double-contact with each $\mathscr{B}_{i}$. To this there corresponds (Section 2.1) a rational sextic curve on $D$ with the $b_{i}$ for bitangents. This was how $\mathscr{S}$ was initially discovered. In fact, there is (Theorem 1) a unique (irreducible) sextic curve with the $b_{i}$ for bitangents: call this $\mathscr{S}$ henceforth. The group of projectivities of $P$ is the symmetric group $\Sigma_{\varsigma}$, and this is the group of $D$. Its subgroup 
$A_{5}$ fixes each half of $\delta$ and thus $\mathscr{S}$. The coset $\Sigma_{5} \backslash A_{5}$ interchanges the halves of $\delta$, so there is (Theorem 1) a unique sextic curve $\hat{\mathscr{S}}$ with the $a_{i}$ for bitangents.

In the course of the proof of the projective equivalence of all non-singular rational sextics with six bitangents, we shall see that such a sextic lies on a unique cubic surface (Theorem 2), which must (Theorem 3) be a diagonal surface with one half of its distinguished double-six for the bitangents. Thus (Theorem 4) rational sextics with six bitangents occur in related pairs on diagonal cubic surfaces, and $\mathscr{S}$ and $\hat{\mathscr{S}}$ are such a related pair. One of the 72 families of twisted cubics on $D$ consists of those having the six $b_{i}$ for chords [1, p. 175], [12, p. 126]. The members of this family $\mathscr{F}$ each meet $\mathscr{S}$ in two points, and there are two members of $\mathscr{F}$ through each Eckardt point that touch $\mathscr{S}$. The 20 such points of contact on $\mathscr{S}$ are (Theorem 1) its intersections with its mate $\hat{\mathscr{S}}$ : the twisted cubics on $D$ touching $\hat{\mathscr{S}}$ at these points and having the $a_{i}$ for chords pass in pairs through the Eckardt points.

1.3 There is a unique quadric, the Schur quadric $Q$ of $\delta$, that has the $a_{i}, b_{i}$ for six pairs of polar lines [1, p. 161], [10, p. 147]. $Q$ meets the $b_{i}$ in their points of contact with $\mathscr{S}$, and the $a_{i}$ in their points of contact with $\hat{\mathscr{S}}$ (Section 2.1). There is a pencil of curves on $D$ which have 3-point (at least) contact with $Q$ at each of its 12 intersections with the six $b_{i}$. All but four of the curves of this pencil are irreducible non-singular 18-ics of genus 10. The four exceptional curves are: (i) $\mathscr{S}$ counted thrice; (ii) Bring's sextic that is the intersection of $Q$ and D; (iii) a rational 18-ic with each Eckardt point for double point, and whose tangents at each Eckardt point are those of the two members of $\mathscr{F}$ through that point that touch $\mathscr{S}$; (iv) the set of six twisted cubics of $\mathscr{F}$ that pass through the contact points of $\mathscr{S}$ with the six $b_{i}$. (Section 2.3). Bring's curve is the canonical sextic of genus 4 that has the largest collineation group [5, p. 539]. Because it is symmetrically related to $\mathscr{S}$ and $\hat{\mathscr{S}}$ we see that Bring's curve has 12 of the twisted cubics on $D$ touching it inflexionally twice (Section 2.3).

1.4. As we have already mentioned some of our arguments are valid if we treat the six bitangents as quadrisecants. They show (Theorem 3 ) that any space sextic curve with six or more quadrisecants is either a $(4,2)$ or a $(5,1)$ curve on a quadric, or is on a unique non-singular cubic surface and has the lines of one half of one of the doublesixes for its quadrisecants.

\section{The pair of non-singular sextics with six bitangents on a diagonal cubic surface}

2.1 Let $D$ be a diagonal cubic surface. We take its representation in a plane $\pi$ as described in Section 1.2. We shall make use several times of the fact [1, p. 191], $[12, \mathrm{p} .124]$ that to an irreducible curve of order $n$ in $\pi$ which has the base points $A_{1}, A_{2}, \ldots, A_{6}$ as points of respective multiplicities $\lambda_{1}, \lambda_{2}, \ldots i_{6}$ there corresponds an irreducible curve on $D$ of order

$$
3 n-\lambda_{1}-\lambda_{2}-\lambda_{3}-\lambda_{4}-\lambda_{5}-i_{6}
$$

here we allow the possibility $i_{i}=0$. 
All hexagons with 10 Brianchon points are projectively equivalent [3, p. 275]. Such a Clebsch hexagon $H$ has associated with it a non-singular conic $\mathscr{C}$ that has [3, p. 283] double-contact with the conic through the vertices of $H$ other than $A_{i}$. Thus $\mathscr{C}$ has double contact with all the $\mathscr{B}_{i}$. Moreover [3, p. 281] $\mathscr{C}$ contains none of the $A_{i}$. Hence, by (3) and (2), $\mathscr{C}$ corresponds to a sextic curve $\mathscr{S}$ on $D$ with each $b_{i}$ for bitangent. As $\mathscr{C}$ contains no $A_{i}$ the correspondence from $\mathscr{C}$ to $\mathscr{S}$ is biregular on their sets of points. Hence $\mathscr{S}$ is non-singular and rational with $\mathscr{C}$. One could, alternatively, note that through any point of $\mathscr{C}$ there pass cubic curves of $\pi$ through all the $A_{i}$ that do not touch $\mathscr{C}$ at this point; correspondingly there are planes through any point of $\mathscr{P}$ having intersection multiplicity 1 at that point.

The points of contact of $\mathscr{C}$ and $\mathscr{B}_{i}$, say $U_{i}, V_{i}$, are the points of contact of $\mathscr{B}_{i}$, and $\mathscr{C}$, with their tangents $u_{i}, v_{i}$ from $A_{i}[3$, p. 283], $[4, \S 1.5]$. The composite cubic curve consisting of $u_{i}$ and $\mathscr{B}_{i}$ meets $\mathscr{C}$ four times at $U_{i}$ and twice at $V_{i}$. This is a cubic through the $A_{i}$. It corresponds to a plane meeting $\mathscr{S}$ four times at a contact with $b_{i}$. The conics that are the residual intersections of $D$ with the planes through $b_{i}$ cut $b_{i}$ in pairs of points of an involution whose double points are parabolic points of $D[1, \mathrm{p} .211],[10$, p. 149]. Such conics correspond in $\pi$ to the lines through $A_{i}$ (since their planes correspond to cubics through the $A_{i}$ with $\mathscr{B}_{i}$ for a component) which cut $\mathscr{C}$ in an involution with $U_{i}, V_{i}$ for double points. Thus the points of contact of $\mathscr{S}$ with its bitangents are stalls of $\mathscr{S}$ and parabolic points on $D$. Thus they are on the Schur quadric $Q$ of $\delta[10$, p. 151].

2.2. It is time to go beyond the straightforward translation of known facts in $\pi$ and to prove

Theorem 1. Let $D$ be a diagonal cubic surface with pentahedron $P$. Let $\delta$ be the distinguished double-six on $D$ whose halves are the six lines $a_{i}$ and the six lines $b_{j}$, where $a_{i}$ is skew to $b_{j}$. Then:

(i) there is a unique irreducible sextic curve $\mathscr{S}_{a}$ having the lines $a_{i}$ for bitangents, and a unique sextic curve $\mathscr{S}_{b}$ having the lines $b_{j}$ for bitangents;

(ii) $\mathscr{S}_{a}$ and $\mathscr{S}_{b}$ are non-singular rational curves on $D$, and have the diagonals of $P$ for chords;

(iii) the contacts of $\mathscr{S}_{a}\left(\mathscr{S}_{b}\right)$ with the $a_{i}\left(b_{j}\right)$ are all its stalls;

(iv) $\mathscr{S}_{a}$ and $\mathscr{S}_{b}$ have 20 points of intersection, which are the points of contact of $\mathscr{S}_{a}\left(\mathscr{S}_{b}\right)$ with the pairs of twisted cubics on $D$ through the Eckardt points with the $a_{i}\left(b_{j}\right)$ for chords that touch $\mathscr{S}_{a}\left(\mathscr{S}_{b}\right)$.

Proof. (i) We show, first that if $\Gamma$ is a sextic with the $b_{i}$ for bitangents then its correspondent $\Gamma^{*}$ in $\pi$ is a conic. Since $\Gamma$ meets $D$ in, counted with multiplicity, at least 24 points it does lie on $D$, and so is a space sextic! Let $\Sigma_{5}$ be the group of $P$, and let $\rho$ be an involution in $\Sigma_{5} \backslash A_{5}$. Then [6, p. 541] $\rho$ is a harmonic inversion with an Eckardt point for centre. Since the $a_{i}$ are skew at least one of them, say $a_{k}$, is not in the fixed plane of $\rho$ and so is moved by $\rho$ to an intersecting line $c$. Since $\Sigma_{5}$ is [6, p. 540], [9, p. 201] the group of $P$ and $D$, the involution $\rho$ must fix $\delta$. By the intersection properties of (1) $c$ must be a $b_{j}$. Hence $\rho$ interchanges the two halves of $\delta$. Thus $\rho \Gamma$ is a sextic with 
the $a_{i}$ for bitangents. Its correspondent $\mathcal{N}$ in $\pi$ must have $A_{i}$ as a point of multiplicity $\mu_{i} \geq 4$ : at this stage of our argument it is conceivable that a bitangent meets $\rho \Gamma$ in further points. Suppose that $\mathcal{N}$ has order $n$. Then, by (3),

$$
6=3 n-\left(\mu_{1}+\ldots+\mu_{6}\right) \leq 3 n-24
$$

so that $n \geq 10$.

A twisted cubic on $D$ with the $b_{i}$ for chords corresponds [12, pp. 126, 127] to a line of $\pi$ through no $A_{i}$, and a twisted cubic with the $a_{i}$ for chords to a quintic curve with the $A_{i}$ for double points. Since $\rho$ interchanges these two types of twisted cubic its correspondent $\rho^{*}$ in $\pi$ is a Cremona transformation with quintic homaloids. Also, $\rho^{*}$ takes the set of fundamental points $A_{i}$ to the set of conics $\mathscr{B}_{j}$. Hence $\rho^{*} \mathscr{N}$ has order $5 n$ and contains $\mathscr{B}_{i}$ as a component of multiplicity $\mu_{i}$. The residual of these components, which is $\Gamma^{*}$, has order

$$
5 n-2\left(\mu_{1}+\ldots+\mu_{6}\right)=12-n
$$

by (4). Since lines in $\pi$ correspond to lines, conics or twisted cubics on $D[12$, pp. 125,126 , we must have $n \leq 10$. It follows that $n=10$ and that $\Gamma^{*}$ has order 2 ; as claimed.

$\Gamma^{*}$ has double contact with the $\mathscr{B}_{i}$. We now show that it must be $\mathscr{C}$. Since it is possible for a set of conics to all have double contact with a pair of conics it is inevitable that we must use the special geometric features of $H$ (see Section 1.2). If $\Gamma^{*}(x)$ is a quadratic form of $\Gamma^{*}$ then there is a linear form $L_{i}^{*}(x)$ such that $\mathscr{B}_{i}$ has for a quadratic form

$$
\mathscr{B}_{i}(\underline{x})=\Gamma^{*}(\underline{x})+\left[L_{i}(\underline{x})\right]^{2}
$$

If we subtract, for $j \neq i, \mathscr{B}_{j}(\underset{\sim}{x})$ from $\mathscr{B}_{i}(\underset{\sim}{x})$ we see that $\left[L_{i}(\underset{x}{x}]^{2}-\left[L_{j}(\underline{x})\right]^{2}=0\right.$ is a line pair of the pencil containing $\mathscr{B}_{i}$ and $\mathscr{B}_{j}$. Since these conics meet in the four vertices of $H$ other than $A_{i}, A_{j}$, this is a genuine line pair, and the lines $L_{i}, L_{j}$ corresponding to $L_{i}(\underline{x})$ and $L_{j}(x)$ are distinct and meet in a diagonal point of the quadrangle $\mathscr{B}_{i} \cap \mathscr{B}_{j}$.

Co-ordinates may be chosen so that the vertices of $H$ are $(1, \pm j, 0),(0,1, \pm j)$ and $( \pm j, 0,1)$ where $j^{2}=j+1[3, \mathrm{p} .274]$. The quadrangle with the first four of these for vertices has for diagonal points $(0,1,0)$ and $\left(1,0, \pm j^{2}\right)$. The last two are [3, p. 274] the two Brianchon points on the edge of $H$ joining $( \pm j, 0,1)$, and the first is the pole with respect to $\mathscr{C}$, which has quadratic form $x^{2}+y^{2}+z^{2}[3$, p. 277], of that edge. Since the group of $H$ is transitive on its edges [3, p. 278] we see that the diagonal points of the quadrangle not having $A_{i}, A_{j}$ for vertices are the pole of $A_{i} A_{j}$ with respect to $\mathscr{C}$ and the two Brianchon points on $A_{i} A_{j}$.

The diagonal points of the five quadrangles not having $A_{1}$ for a vertex have 15 distinct diagonal points, namely the pairs of Brianchon points on the lines $A_{1} \boldsymbol{A}_{i}(i>1)$ and their poles. Thus $L_{1}$ meets $L_{2}, \ldots, L_{6}$ in five of these distinct points. Since no three 
Brianchon points are collinear [3, p. 285], at least three of these points on $L_{1}$ are poles of edges of $H$ through $A_{1}$. Hence $L_{1}$ is the polar line of $A_{1}$ with respect to $\mathscr{C}$. This (see Section 2.1) is the line of contact of $\mathscr{C}$ and $\mathscr{B}_{1}$. There are corresponding results for all the $L_{i}$. Hence there are $\alpha_{i}, \beta_{i}$ such that

$$
\mathscr{B}_{i}(x)=\alpha_{i} \mathscr{C}(\underline{x})+\beta_{i}\left[L_{i}(x)\right]^{2}
$$

where $\mathscr{C}(\underline{x})$ is a form corresponding to $\mathscr{C}$. Hence, from (5),

$$
\Gamma^{*}(\underline{x})-\alpha_{i} \mathscr{C}(\underline{x})=\left(\beta_{i}-1\right)\left[L_{i}(\underline{x})\right]^{2}
$$

If no $\beta_{i}$ is 1 then $\Gamma^{*}$ and $\mathscr{C}$ are distinct conics which determine a pencil containing six repeated lines. Since a pencil of conics that contains a non-singular member has at most four singular members [4, pp. 177-183] this is impossible. Hence some $\beta_{i}$ is 1 . Then, from $(6), \Gamma^{*}(\underline{x})$ is a multiple of $\mathscr{C}(\underline{x})$ so that $\Gamma^{*}$ is $\mathscr{C}$, as required. Since the lines $A_{i} A_{j}$ meet $\mathscr{C}$ in pairs of points and correspond [12, p. 125] to the diagonals of $P$, the discussion of $\mathscr{S}$ in Section 2.1 gives (i) and (ii). Also (iii) follows from Section 2.1 and the fact (Section 1.1) that a non-singular rational sextic has 12 stalls.

(iv) We have already noted that the twisted cubics of the family with the $b_{i}$ for chords correspond in $\pi$ to the lines through no $A_{i}$. The lines through a Brianchon point cut an involution on $\mathscr{C}$ and two of them touch $\mathscr{C}$. The 20 points of contact are the 20 intersections of $\mathscr{C}$ with 10 -ic $\rho^{*} \mathscr{C}[4, \S 4.5]$ which is the correspondent of $\mathscr{S}_{a}$ (see above). Translation of these results to $D$ and the use of $\rho$ now gives (iv), and the proof is complete.

2.3. It was shown in [4, Theorems 1,3$]$ that Bring's curve, the intersection of $D$ with the Schur quadric $Q$ of $\delta$, corresponds in $\pi$ to the unique sextic curve $S$ with the $A_{i}$ for double points and $u_{i}, v_{i}$ for nodal tangents. Further [4, Theorem 1] $S$ has 12 inflexions which are the six pairs of points $U_{i}, V_{i}$ on $\mathscr{C}$ and the line $U_{i} V_{i}$ is the inflexional tangent to $S$ at $U_{i}$ and $V_{i}$. The sextics meeting each $U_{i} V_{i}$ three times (at least) at $U_{i}$ and $V_{i}$ form a pencil $\mathscr{P}$ [4, Theorem 2] whose only reducible members are the conic $\mathscr{C}$ counted thrice and the set of six lines $U_{i} V_{i}$. All other members have $U_{i} V_{i}$ for proper biflexional tangents. The member of $\mathscr{P}$ through the Brianchon points is rational and has these points for double points with the tangents to $\mathscr{C}$ from the Brianchon points as nodal tangents. Apart from this curve, the reducible curves, and $S$, the members of $\mathscr{P}$ are non-singular and of genus 10 . That $Q$ has 3 point intersection with Bring's curve at its intersections with the lines of $\delta$ was proved in $[6, p .543]$. Translation to $D$ using information accumulated during the proof of Theorem 1 establishes the results stated in Section 1.3. 


\section{Space sextics with six bitangents}

3.1. Let $\Gamma$ now be an irreducible space sextic curve. For precision, we remark that by a bitangent of $\Gamma$ we mean a line that is a tangent to $\Gamma$ at at least two distinct points; this includes the possibility that it is a nodal tangent at a singular point. This is a special type of quadrisecant, namely a line with four, at least, intersections with $\Gamma$ when we count the order of its contact at the intersections. We note that two quadrisecants of $\Gamma$ are skew; else their plane would have, counting multiplicities, at least 8 intersections with $\Gamma$.

3.2. A cubic surface containing $\Gamma$ must, since it meets any quadrisecant in four points or more, contain every quadrisecant of $\Gamma$. We need

Proposition 1. If $\Gamma$ has at least six quadrisecants then it cannot lie on a singular irreducible cubic surface.

Proof. Suppose that $\Gamma$ lies on such a surface $C$. A cubic surface with a triple point is a cone [13, p. 392], and all its lines concur at its vertex; it thus cannot be $C$. If $C$ is ruled then $[5$, p. 15], $[12$, pp. $65,136,206]$ each of its generators meets $\Gamma$ in the same number of points, say $m$. Since $C$ contains at most two non-generator lines [5, pp. 32, 33], [11, p. 164], [12, p. 135], [13, p. 382], at least four generators must be quadrisecants of $\Gamma$; so that $m \geq 4$ and thus each generator is a quadrisecant. But through a general point of the directrix (double) line $d$ on $C$ pass two generators [5, p. 8], [12, p. 135], $[13$, p. 384]: for Cayley's cubic one of these is $d$. We have a contradiction to the final sentence of Section 3.1.

Thus $C$ must be one of the 21 types of cubic surface with isolated double points [8,pp. 63-82], [9, p. 222], [11, p. 170]. Each of these contains a finite number of lines. Let $l_{i}, i=1,2, \ldots, 6$, be six quadrisecants of $\Gamma$. Any line meeting four of these meets $C$ in at least four distinct points, and so is on $C$. Through any line on $C$ there pass at most five planes whose residual intersection with $C$ is a reducible conic [13, pp. 391, 392]. Thus no line meets all six $l_{i}$. The five $l_{i}$ other than a particular $l_{j}$ may have a common transversal; if they do we shall let $m_{j}$ denote one such transversal. If $m_{1}, m_{2}, \ldots, m_{5}$ exist then $C$ has at least 11 lines. Suppose that just $m_{1}, m_{2}, m_{3}, m_{4}$ occur. Then $l_{1}, l_{2}, l_{3}, l_{4}$ have at least one transversal, which cannot be $m_{1}, m_{2}, m_{3}, m_{4}$ else all six $l_{i}$ would have a common transversal. Again, $C$ has at least 11 lines. By considering similar 4-subsets of the $l_{i}$, we see that if there are $0,1,2$ or $3 m_{i}$ then $C$ has at least $21,17,14,12$ lines respectively. Only five types of the possible singular cubic surfaces have 11 or more lines. These are [9, p. 220], [11, p. 164] those whose sets of singular points are $C^{2}, 2 C^{2}, B^{3}, 3 C^{2}$ and $B^{3}+C^{2}$ respectively, where $C^{2}$ denotes a conic node and $B^{3}$ an ordinary binode. These cubics contain $21,16,15,12,11$ lines respectively. In the last case 8 of these 11 lines are the join $B^{3} C^{2}$, four other lines through $C^{2}$ and three other lines through $B^{3}[8$, p. 73$]$, so there is no possibility of six skew lines on this cubic. The other four types may be represented by plane cubic curves with six distinct base points $B_{1}, \ldots, B_{6}$. But these base points are not in general position. For the cubic with 21 lines the $B_{i}$ lie on a conic; for the cubic with 16 lines $B_{2}, B_{3}, B_{4}$ and $B_{2}, B_{5}, B_{6}$ are collinear triads with $B_{1}$ general; for the cubic with 15 lines $B_{1}, B_{2}, B_{3}$ and 
$B_{4}, B_{5}, B_{6}$ are collinear triads; and for the cubic with 12 lines $B_{1}, B_{2}, B_{3} ; B_{1}, B_{5}, B_{6}$; $B_{3}, B_{4}, B_{5}$ are collinear triads [7, pp. 640-644], [12, pp. 143, 144]. The triads, and in the first case the conic through the $B_{i}$; correspond to the double points. The lines on $C$ correspond to the neighbourhoods of the $B_{i}$, any conics through five but not six of the $B_{i}$, and the joins $B_{i} B_{j}$ where $B_{i}, B_{j}$ are not part of a triad. It is easy to check that in each case it is impossible to find six skew lines on $C$. Alternatively, one can check this from Henderson's equations for the lines on $C$ which are given in simple co-ordinate form in [8, pp. 6-82]. We have our required contradiction.

We can now prove

Theorem 2. An irreducible space sextic curve with at least six distinct quadrisecants is either

(i) a (4,2) or a (5,1) curve on a quadric surface, in which case it has infinitely many quadrisecants,

or

(ii) a rational non-singular sextic that lies on a unique and non-singular cubic surface; in which case its quadrisecants are the six lines of one half of a double-six on the cubic surface, and it is skew to the lines of the complementary half.

Proof. Since a quaternary cubic form has 20 coefficients there is a cubic surface $C$ through 19 points of such a curve $\Gamma$. By Bezout's Theorem, $C$ contains $\Gamma$. If $C$ is reducible then it must have a quadric component that contains $\Gamma$. This quadric contains the skew quadrisecants and so is not a cone. Hence the quadrisecants belong to one regulus, and $\Gamma$ must be a $(5,1)$ or a $(4,2)$ curve.

So, suppose that $C$ is irreducible. Then, by Proposition 1 , it must be non-singular. Any other cubic surface containing $\Gamma$ also contains six quadrisecants of $\Gamma$ and so would meet $C$ in a composite curve of order at least 12; an impossibility. The only sets of six skew lines on a non-singular cubic surface are the 72 halves of its 36 double-sixes, and there are no sets of seven skew lines [7, p. 485]. Take the plane representation $\pi$ of $C$ (see Section 1.2) so that the quadrisecants of $\Gamma$ correspond to the neighbourhoods of the six base points $A_{i}[7$, p. 489], [12, pp. 125, 126]. Let $\Gamma$ correspond to the curve $\Gamma^{*}$ in $\pi$ of order $n$. Suppose that $\Gamma^{*}$ has multiplicity $\mu_{i}$ at $A_{i}$. Then we again have (4) with $\mu_{i} \geq 4$, and thus $n \geq 10$. Since $\Gamma$ is irreducible we must have $\mu_{i}<6$. Thus the only possibilities for (4) are:

(i) each $\mu_{i}$ is 4 , and $n=10$;

(ii) three $\mu_{i}$ are 4 , the others are 5 , and $n=11$;

(iii) each $\mu_{i}$ is 5 , and $n=12$.

The deficiency $D$ of $\Gamma^{*}$ is non-negative. Thus, since $\Gamma^{*}$ may have multiple points other than the $A_{i}$ we must have $[12$, p. 30],

$$
(n-1)(n-2)-\Sigma_{i=1}^{6} \mu_{i}\left(\mu_{i}-1\right) \geq 0 .
$$

Neither possibility (ii) nor possibility (iii) satisfies this inequality. Thus $\Gamma^{*}$ is a 10 -ic 
with the $A_{i}$ for exactly 4 -tuple points. The conic through $A_{1}, A_{2}, A_{3}, A_{4}, A_{5}$ corresponds to the line $b_{6}$ of the complementary half of the double-six. Since the sum of its multiplicities of intersections with $\Gamma^{*}$ at the $A_{i}$ is at least $5 \times 4=20$, it meets $\Gamma^{*}$ nowhere else. Thus $b_{6}$ is skew to $\Gamma$. Similar statements holds for $b_{1}, \ldots, b_{5}$.

Now take the plane representation of $C$ having base points $B_{1}, \ldots, B_{6}$ corresponding to the lines $b_{i}$. Then the quadrisecants correspond to conics through five of the $B_{i}$. And $\Gamma$ corresponds to a curve $\Gamma^{\dagger}$ not through any $B_{i}$. Hence, by (3), $\Gamma^{\dagger}$ has order 2 . Thus, as argued for $\mathscr{S}$ in Section $2.1, \Gamma$ is rational and non-singular. We are done

We should remark that we could use the deficiency inequality argument in place of the discussion involving $\rho^{*}$ to show, when proving Theorem 1 , that the $\mathcal{N}$ of Section 2.2 has order 10 . It seems worthwhile, however, to show the efficient effectiveness of both approaches.

3.3 It is time to return to the bitangent situation. We have

Theorem 3. An irreducible sextic curve with at least six distinct bitangents has exactly six bitangents, and is either

(i) a $(4,2)$ curve on a quadric

or

(ii) a rational non-singular sextic lying on a diagonal cubic surface $D$, and having one half of the distinguished double-six of $D$ for its bitangents.

Proof. For an $(\alpha, \beta)$ curve on a quadric there are, counting multiplicities, $2 \beta(\alpha-1)$ points $R$ such that the $\alpha$ generator through $R$ has more than simple intersection with the curve at $R[5$, p. 10$],[12$, p. 60$]$. For a $(5,1)$ curve this number is 8 , and so the curve can have at most four bitangents. One can also see this by taking the stereographic projection of the quadric from a point on the curve. For a $(4,2)$ curve the number is 12. Hence there can be at most six bitangents. We verify in Section 3.4 below that such curves do exist.

By Theorem 2, we must show that (ii) holds for a sextic $\Gamma$ with six bitangents that lies on a non-singular cubic surface $C$. We return to the plane representation of $\Gamma$ given by the last paragraph of the proof of Theorem 2 . Now the conic $\Gamma^{\dagger}$ has double contact with the conic $\mathscr{B}_{i}$ through the five $B_{j}$ other than $B_{i}$.

We may take co-ordinates $\mathrm{x}=(x, y, z)$ in $\pi$ such that $B_{1}, B_{2}, B_{3}$ are $(1,0,0),(0,1,0)$, $(0,0,1)$ respectively. Since $\mathscr{B}_{4}$ and $\mathscr{B}_{5}$ do not touch at $B_{i}(i \leq 3)$, this point cannot be a contact of either with $\Gamma^{\dagger}$, and so is not on $\Gamma^{\dagger}$. By replacing $x, y, z$ by suitable multiples we may thus take for a quadratic form of $\Gamma^{\dagger}$

$$
Q(\mathbf{x})=x^{2}+y^{2}+z^{2}+2 f y z+2 g z x+2 h x y .
$$

Suppose that $\mathscr{B}_{i}$ has for a quadratic form

$$
B_{i}(\mathbf{x})=Q(\mathbf{x})-\left[L_{i}(\mathbf{x})\right]^{2},
$$


where $L_{i}(\mathbf{x})$ corresponds to the line of contact $L_{i}$ with $\Gamma^{\dagger}$. Since $\mathscr{B}_{4}, \mathscr{B}_{5}, \mathscr{B}_{6}$ contain $X, Y, Z$ their forms do not contain non-zero $x^{2}, y^{2}, z^{2}$ terms. Hence each of $L_{4}(\mathbf{x}), L_{5}(\mathbf{x})$, $L_{6}(\mathbf{x})$ is one of $\pm(x+y+z), \pm(-x+y+z), \pm(x-y+z), \pm(x+y-z)$. By replacing some of $x, y, z$ by their negatives, if necessary, we may assume that $\pm(x+y+z)$ does not occur, and then by renumbering the $B_{4}, B_{5}, B_{6}$ we may, since their sign's are immaterial, assume that

$$
L_{4}(\mathbf{x})=-x+y+z ; L_{5}(\mathbf{x})=x-y+z ; L_{6}(\mathbf{x})=x+y-z
$$

The points common to $\mathscr{B}_{4}$ and $\mathscr{B}_{5}$ satisfy, by (8), $L_{4}(\mathbf{x})= \pm L_{5}(\mathbf{x})$. The negative sign yields points with $z=0$; these must be $B_{1}$ and $B_{2}$. The positive sign yields points with $x=y$. One of these is $B_{3}$. The other must be $B_{6}$ with co-ordinates $(1,1, \gamma)$ for some $\gamma \neq 0$. Similarly, $B_{4}$ is $(\alpha, 1,1)$ and $B_{5}$ is $(1, \beta, 1)$ for some $\alpha, \beta$ not 0 . Thus the lines $B_{1} B_{4}, B_{2} B_{5}, B_{3} B_{6}$ concur at $U=(1,1,1)$. Since no three $B_{i}$ are collinear $U$ is not one of these; none of $\alpha, \beta, \gamma$ is 1 . We see from (7), (8) and (9) that

$$
2+2 f+2 g \alpha+2 h \alpha=0,
$$

with corresponding equations for $\beta, \gamma$.

Since $\mathscr{B}_{1}$ contains $B_{2}$ and $B_{3}$ we have, by (7), (8),

$$
L_{1}(\mathbf{x})= \pm(y \pm z+p x)
$$

for some $p$. Since $\mathscr{B}_{1}$ contains $B_{4}$ we also see that

$$
2+\alpha^{2}+2 f+2 g \alpha+2 h \alpha=(1 \pm 1+p \alpha)^{2}
$$

which, by (10), becomes

$$
\alpha^{2}=(1 \pm 1+p \alpha)^{2}
$$

On demanding that $B_{5}$ and $B_{6}$ lie on $\mathscr{B}_{1}$ we similarly obtain

$$
\begin{aligned}
& \beta^{2}=(\beta \pm 1+p)^{2} \\
& \gamma^{2}=(1 \pm \gamma+p)^{2}
\end{aligned}
$$

The lower sign possibility yields $p^{2}=1$ from (12). Then (11), (9), (8) would give $\mathscr{B}_{1}$ is $\mathscr{B}_{5}$ or $\mathscr{B}_{6}$ a contradiction. Hence, the upper sign possibility must occur, so, from (13), (14), $\beta$ and $\gamma$ both satisfy

$$
(1+p)(1+p+2 t)=0
$$


We cannot have $p=-1$, else, by (12), $\alpha=1$. Hence, from (15), $\beta=\gamma$. Similarly, by considering $\mathscr{B}_{2}$ and $\mathscr{B}_{3}$, we see that $\alpha=\beta=\gamma$.

It follows that $B_{1} B_{5}, B_{2} B_{4}$ and $B_{3} B_{6}$ concur at $\left(1,1, \alpha^{-1}\right) ; B_{1} B_{4}, B_{2} B_{6}$ and $B_{3} B_{5}$ at $\left(\alpha^{-1}, 1,1\right)$; and $B_{1} B_{6}, B_{2} B_{5}, B_{3} B_{4}$ at $\left(1, \alpha^{-1}, 1\right)$. Together with $U$ these are four centres of perspective of the triangles $B_{1} B_{2} B_{3}, B_{4} B_{5} B_{6}$, and are Brianchon points of the hexagon $H$ with the $B_{i}$ for vertices.

It follows that each of the 10 pairs of triangles, whose vertices are those of $H$, has at least four centres of perspective, each a Brianchon point. Since exactly three edges of $H$ pass through any Brianchon point, it is the centre of perspective of exactly four such pairs of triangles. Hence $H$ has at least $10 \times 4 / 4=10$ Brianchon points. Since [3, p. 275] a hexagon can have at most 10 Brianchon points, $H$ has exactly 10 . Thus [4, Section 4.2] (see [9, pp. 197, 199, 201]) $C$ is a diagonal cubic surface $D$ with the $b_{i}$ forming one half of its distinguished double-six $\delta$. We may note that the 15 joins $B_{i} B_{j}$, three through each Brianchon point, correspond to the diagonals of the pentrahedron $P$ of $D$, three through each Eckardt point. The proof is complete.

Since any two pentahedra are projectively equivalent, so are any two diagonal cubic surfaces. The group $\Sigma_{5}$ of $D$ has elements interchanging the halves of $\delta$. Any nonsingular $(4,2)$ quadric curve has genus 3 (Section 1.1). Hence, from Theorem 1 we obtain

Theorem 4. Non-singular rational space sextic curves with six bitangents are projectively equivalent, have the alternating group $A_{5}$ for collineation group, and lie by pairs on diagonal cubic surfaces.

3.4. We ought to exhibit a non-singular $(4,2)$ curve on a quadric with six bitangents. Take co-ordinates so that the quadric $Q$ is parametrised as $\left(x_{0}, x_{1}, x_{2}, x_{3}\right)=(\lambda \mu, \lambda, \mu, 1)$. Let

$$
F(\lambda, \mu)=\lambda^{4}+\lambda^{3} \mu^{2}-8^{-1} \lambda+(64)^{-1} \mu^{2}
$$

Then $F(\lambda, \mu)=0$ corresponds to a sextic curve $\Gamma$ on $Q$. To each value of $\mu$, including $\infty$, correspond four $\lambda$, and to each value of $\lambda$ correspond two of $\mu$. Thus $\Gamma$ is a $(4,2)$ curve. The generator $\mu=\infty$ though $(1,0,0,0)$ meets $\Gamma$ in the four distinct points given by $\lambda=\infty, 64 \lambda^{3}=1$. The generator $\lambda=\infty$ 'touches' $\Gamma$ at $(1,0,0,0)$. The projection $\Gamma^{*}$ of $\Gamma$ from $(1,0,0,0)$ onto $x_{0}=0$ is a quintic curve with $(0,0,1,0)$ as an ordinary triple point and $(0,1,0,0)$ as a simple point. It is easy to check that $(0,0,1,0)$ is the only singular point of $\Gamma^{*}$. Thus $\Gamma^{*}$, and hence $\Gamma$, has $[12$, pp. 30, 53] genus

$$
g=\frac{1}{2} \cdot 4 \cdot 3-\frac{1}{2} \cdot 3 \cdot 2=3 \text {, }
$$

and $\Gamma$ is non-singular. 
Suppose that $\mu^{6}=1$. Define $\alpha, \beta$ by

$$
2(\alpha+\beta)=-\mu^{2}, \quad 8 \alpha \beta=-\mu^{4} .
$$

Then $\alpha \neq \beta$. Direct checking shows that $F(\lambda, \mu)$ is the square of $(\lambda-\alpha)(\lambda-\beta)$. Hence the generator corresponding to this $\mu$ touches $\Gamma$ at two distinct points. The six possible such $\mu$ give six bitangents for $\Gamma$.

3.5. We conclude by giving a heuristic argument that suggests there are many classes of $(4,2)$ curves on $Q$ with six bitangents. A quartic surface $F$ has 35 coefficients in a corresponding quartic form. Take $F$ to contain two skew generators on $Q$. This imposes 10 conditions on the coefficients, and $F$ meets $Q$ residually in a $(4,2)$ curve. Take six other generators of the same regulus, take two distinct points on each of the first five, and one point $L$ on the last line $l$. The demand that $F$ touches these generators at these 11 points imposes 22 further linear conditions. We expect to have $F$ belonging to an $\infty^{2}$ system. This system contains the repeated quadric $Q^{2}$. Hence it should cut $l$ residually to the repeated point $L$ in an involution. One of the united points will be distinct from $L$. The corresponding $F$ will cut $Q$ residually in a $(4,2)$ curve with the six generators for bitangents.

\section{REFERENCES}

1. H. F. BAKer, Principles of Geometry, Vol III (Cambridge University Press, 1923).

2. H. F. BAKer, Principles of Geometry, Vol V, (Fredrick Ungar Publishing Co, New York, 1960).

3. R. H. Dye, Hexagons, conics, $A_{5}$ and $P S L_{2}(K)$, J. London Math. Soc. (2) 44 (1991), 270286.

4. R. H. DyE, A plane sextic curve of genus 4 with $A_{5}$ for collineation group, J. London Math. Soc. (2) 52 (1995), 97-110.

5. W. L. EdGE, The Theory of Ruled Surfaces, (Cambridge University Press, 1931).

6. W. L. EdGe, Bring's curve, J. London Math. Soc. (2) 18 (1978), 539-545.

7. P. Griffiths and J. Harris, Principles of Algebraic Geometry, (John Wiley and Sons, New York, 1978).

8. A. Henderson, The Twenty-seven lines upon the Cubic Surface (Cambridge University Press, 1911).

9. J. W. P. Hirschfeld, Finite Projective Spaces of Three Dimensions (Clarendon Press, Oxford, 1985).

10. T. G. Room, The Schur quadric of a cubic surface (I), J. London Math. Soc. 7 (1932), 147-154.

11. G. SALmon, Analytical Geometry of Three Dimensions, Vol II (fifth edition) (Chelsea Publ. C, New York, 1965).

12. J. G. Semple and L. Roth, Introduction to Algebraic Geometry, (Clarendon Press, Oxford, 1949). 
13. D. M. Y. Sommer viLLE, Analytical Geometry of Three Dimensions (Cambridge University Press, 1959).

14. J. A. ToDd, Projective and Analytical Geometry (Pitmans, London, 1960).

Department of Mathematics

UNIVERSITY OF NEWCASTLE

NEWCASTLE UPON TYNE NEl 7RU

ENGLAND 